\title{
Inactivation of Prion and Entotoxins by Nitrogen Gas Plasma Exposure
}

\section{Hideharu Shintani*}

Chuo Univertsity, Bunkyo-ku Tokyo 112-8551, Japan

\begin{abstract}
Several inactivation procedures to prion and endotoxins are reported so far. Most of these methods are not applicable to re-usable medical devices due to failure of achievement of material and functional compatibility. Gas plasma inactivation procedure for prion and endotoxin was studied and attain both sterility assurance level (SAL) of $10^{-6}$ and material and functional compatibility in ease.
\end{abstract}

\section{Introduction}

"About what prion is" will be discussed elsewhere, so in this paper we will discuss about inactivation of prion, especially the specific procedure to attain both $10^{-6}$ sterility assurance level (SAL) and material and functional compatibility, which sterilization validation and good manufacturing practices (GMP) require. Normal and abnormal prion are presented in Figure 1 (McDonnell, 2007).Abnormal prion, $\operatorname{PrP}^{\mathrm{sc}}$, is more abundant $\beta$ sheet structure and less $\alpha$ helix structure than normal prion, $\operatorname{PrP}^{c}$. In addition, abnormal prion is quite tolerable to protein digestion enzymes.

Abnormal prion, $\mathrm{PrPsc}$, is a major cause of iatrogenic disease of brain surgery. As a typical example of an iatrogenic disease, prions have been transmitted via instruments used for neurosurgical procedures, from corneal implants and from pituitary growth hormone. The infectious agents are probably small protein particles, which are highly resistant to heat, ethylene oxide gas, glutaraldehyde and formaldehyde and so on [1]. High concentrations of sodium hypochorite $(20,000 \mathrm{ppm}$, average $\mathrm{Cl}$ ) for $1 \mathrm{~h}$ are effective but are corrosive to most medical devices. Moist heat sterilization at $134-138^{\circ} \mathrm{C}$ for $18 \mathrm{~min}$ or $132^{\circ} \mathrm{C}$ for $1 \mathrm{~h}$ is conducted. These procedures are effective against all strains and prions, but material and functional compatibility is not achieved. Other effective procedures to prions include autoclaving at $121.1^{\circ} \mathrm{C}$ for $30 \mathrm{~min}$ in the presence of $1 \mathrm{~N}$ sodium hydroxide $(\mathrm{NaOH})$, autoclaving at $134^{\circ} \mathrm{C}$ for $18 \mathrm{~min}$ in the presence of $1 \mathrm{~N} \mathrm{NaOH}$, soaking in $2.0 \%$ sodium hypochlorite for 60 min, $1 \mathrm{~N} \mathrm{NaOH}$ for $60 \mathrm{~min}, 4 \mathrm{M}$ guanidine thicyanate, or $0.9 \%$ phenolic $(\mathrm{LpH})$ for $30 \mathrm{~min}$. These highly corrosive procedures can cause significant damage to surgical instruments and therefore, are rarely used. Thorough cleaning is of great importance, owing to the doubts on the effectiveness of inactivation methods and the probable variation in the resistance of different prions to heat and disinfectants.

Prions are highly resistant to most physical and chemical agents, with even greater resistance than bacterial spores in some cases (Table 1) [2,3], thus

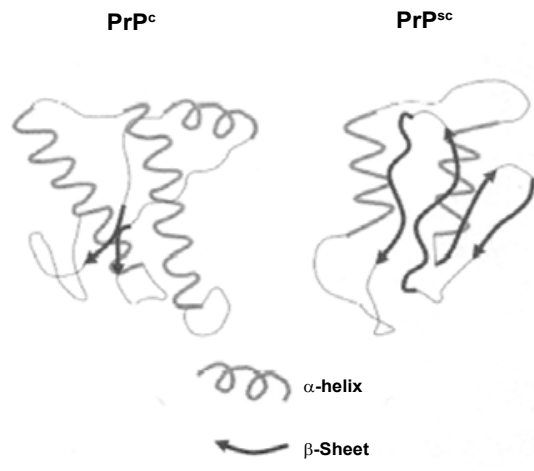

Figure 1: Proposed structure of PrPc and PtPsc.

\begin{tabular}{|l|}
\hline Prions \\
\hline Bacterial Spores \\
\hline Mycobacteria \\
\hline Non-Enveloped Viruses \\
\hline Fungi \\
\hline Vegetative Bacteria \\
\hline Enveloped Viruses \\
\hline
\end{tabular}

Enveloped Viruses

Table 1: Decreasing Order of Resistance of Microorganisms to Disinfectants and Sterilants.

unconventional agents are believed to be highly resistant to many chemical disinfection and physical sterilization processes, including ultraviolet and ionizing radiations, high temperatures. It is little known at present about the mechanisms of inactivation of, or mechanisms of resistance by these unconventional agents.

It is important to note that crude preparations (brain homogenates from infected animals) have been used to investigate the efficacies of various biocides and biocidal processes against prions. The presence of extraneous materials (particularly a high concentration of lipid associated with brain tissue) could, at least to some extent, mask the true efficacies of these processes against the infectious agent. For disinfection of these crude extracts, there is currently no known decontamination procedure that can guarantee the complete absence of infectivity in prion-infected tissues. The most effective process is boiling or superheating under pressure of the tissues in concentrated solutions of sodium hydroxide ( 1 to $2 \mathrm{~N}$ ), which over time can totally dissolve any proteins present. In contrast, prions can survive harsh acid treatment. Formaldehyde, unbuffered glutaraldehyde (acidic $\mathrm{pH}$ ), and ethylene oxide gas have little effect on infectivity, although chlorine-releasing agents (especially hypochlorites), sodium hydroxide, some phenols, and guanidine thiocyanate are more effective. Lower concentrations of hydroxides $(\mathrm{NaOH}$ and $\mathrm{KOH})$ and hypochlorides have been shown to be effective against surface prion contamination, in combination with surfactants and other formulation effects. Extended steam sterilization is effective, although hydration of the prion-infected material appears to be important for optimal inactivation of prions. Further research is required on developing formulations and processes

*Corresponding author: Hideharu Shintani, 1-10-60, Minamidaira, Hino, Tokyo 191-0041, Japan, Tel: +81425922336; E-mail: shintani@mail.hinocatv.ne.jp

Received October 04, 2012; Accepted October 27, 2012; Published October 30 2012

Citation: Shintani H (2012) Inactivation of Prion and Entotoxins by Nitrogen Gas Plasma Exposure. Pharm Anal Acta 3:177. doi:10.4172/2153-2435.1000177

Copyright: ( $) 2012$ Shintani H. This is an open-access article distributed under the terms of the Creative Commons Attribution License, which permits unrestricted use, distribution, and reproduction in any medium, provided the original author and source are credited. 


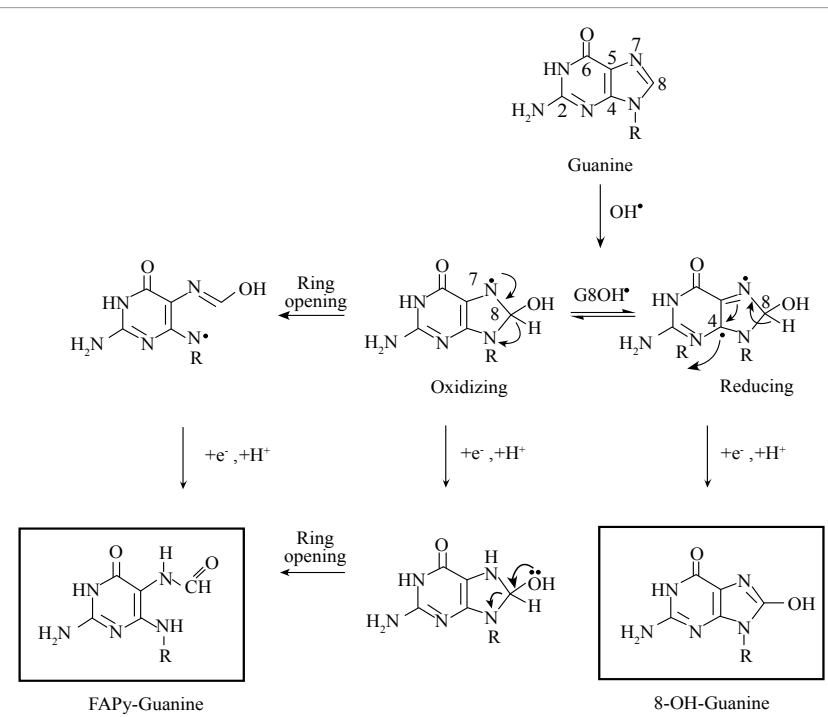

FAPy-Guanine

8-OH-Guanine

Figure 2: Proposed formation pathway from Guanine to $8 \mathrm{OH}$ Guanine by gas plasma exposure.

\section{PrPc Inactivation by LP-TES Method}

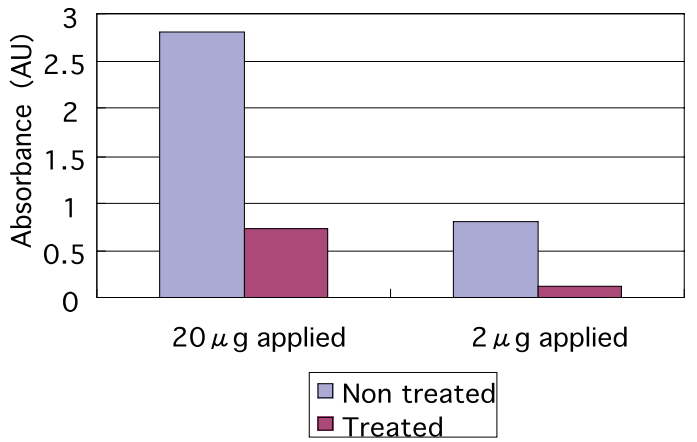

Figure 3: $20 \mu \mathrm{g}, 2 \mu \mathrm{g}$ of PrPc, respectively, was applied. After nitrogen gas plasma exposure, the residue PrPc was sampled with TIA Buffer and determined with ELISA kit from Funakoshi, Tokyo, Japan.

Nitrogen gas plasma exposure condition: [Low pressure method] (Reactor) Anode-Cathode Gap: $40 \mathrm{~mm}$, Vacuum: $43000 \mathrm{~Pa}$, Nitrogen gas flow speed: 6 $\mathrm{L} / \mathrm{min}$, Time: $30 \mathrm{~min}$, Temperature: $60^{\circ} \mathrm{C}$ (Temperature was measured inside of plasma), (Electric Source) Input Power: 84 W, Rep. Freq. 2.5 kHz, Peak Voltage: $19.0 \mathrm{kV}$.

The smaller the prion inoculation, the more degradation is observed. This indicates clump phenomenon inoculated prion onto carrier materials can be formed.

against prions, including the use of oxidizing agents, like gaseous hydrogen peroxide. In case of gaseous hydrogen peroxide exposure, produced $\mathrm{OH}$ radicals attack to amino acids with benzene ring (phenylalanine, tyrosine, tryptophan), resulting in denaturation of prion protein due to failure of maintenance of high-order structure because of incorrect hydrogen bonding between amino acids.

Prions are hydrophobic proteins and have been shown to have affinity for surfaces, including metals and plastics. In most surface disinfection recommendations for use against prions, cleaning is considered a key step to remove most of the contamination prior to chemical or heat inactivation of the prions. However, certain cleaning formulations have been shown to increase the intrinsic resistance of prions or prion-contaminated materials by an unknown mechanism. This has also been observed in treatment with some biocides, including formaldehyde, presumably due to protein fixation. Although prions are considered resistant to proteases, various proteases, including proteinase $\mathrm{K}$ and keratinases, have been shown to degrade prions over time, depending on their concentrations and the exposure conditions.

Prions aggregate to form protein particles or fibrils within various tissues but are predominantly observed within brain tissue. These particles are hydrophobic and are associated with various cellular materials present within the contaminated tissue; for example, brain tissue contains a high concentration of lipid materials. These effects create a penetration challenge for the biocidal process. Various biocides and biocidal processes have been shown to be ineffective against prions. In the case of radiation sterilization, the mechanism of resistance is the absence of any nucleic acid bases associated with prion and endotoxin inactivation [4]. It is concretely speculated due to lack of formation of hydroxylation of DNA and RNA bases such as $8 \mathrm{OHG}$ (8-hydroxy gunanine, Figure 2) [5] to prions and endotoxins, which result in failure of regular hydrogen bonding formation of nucleic acids among Adenine $=$ Tymine $(\mathrm{A}=\mathrm{T})$, Adenine $=$ Uracil $(\mathrm{A}=\mathrm{U})$ and Guanine $\equiv$ Cytosine $(\mathrm{G} \equiv \mathrm{C})$. In addition, radiation sterilization can damage other macromolecules, including proteins due to cleavage of hydrogen bonding to form higher order structures. Biocides that have a cross-linking or fixing mode of action, including alcohols and aldehydes, have also been shown to be ineffective against prions, presumably due to a lack of any degradative effect on the target protein but actual cross-linking with other associated extraneous materials. Biocides or biocidal processes that denature or cause the fragmentation of proteins have been shown to be effective against prions. Moist heat and certain phenolic formulations are proposed to inactivate prions by denaturation. It has been speculated that renaturation of the denatured protein could occur under some conditions, although this has not been observed. Biocides, like sodium hypochlorite, sodium hydroxide, and hydrogen peroxide at high concentrations, appear to cause fragmentation or other structural changes to prions, rendering them noninfectious. It is still possible but considered unlikely that other, as yet unidentified factors are involved in prion infectivity and need to be similarly degraded to ensure complete inactivation.

Almost all inactivation and decontamination procedures to prions reported so far are ineffective. If the procedure is effective to prions, it may cause damage to material and functional compatibility. According to the current sterilization validation and good manufacture practices (GMP), $10^{-6}$ of SAL (sterility assurance level) and material and functional compatibility must be achieved simultaneously. However, the presented procedures for inactivation of prions and endotoxins are failed to satisfy both SAL of $10^{-6}$ and material and functional compatibility simultaneously.

Recently gas plasma sterilization was studied [6,7] and nitrogen gas plasma sterilization can attain both 10-6 SAL and material and functional compatibility in success when applied to prion and endotoxin inactivation. The study example is briefly presented below. The pulsed type nitrogen gas plasma generator is very effective to inactivate prion (Figure 3), bovine serum albumin (BSA, Figure 4), and endotoxin up to $6 \log$ reduction for 30 min exposure (Figure 5) [6] with achievement of SAL of $10^{-6}$ and material and functional compatibility. This is due to the shallow penetration depth of gas plasma sterilization factors such as $\mathrm{NO}$ radicals, $\mathrm{OH}$ radicals, nitrogen metastables and so on at 20 to $40 \mathrm{~nm}$ level (Figure 6) $[6,8]$. This penetration depth is effective to inactivate bioburden incluing bacterial spores. The shallow penetration depth of nitrogen gas plasma exposure compared with other existing sterilization procedures are the most reason why both SAL of $10^{-6}$ and material and functional compatibility simultaneously can easily attain. $\mathrm{OH}$ radicals bind to amino acids, especially amino acids with benzene ring (phenylalanine, tyrosine, tryptophan), resulting in denaturation of prion protein due to failure of maintenance of high-order structure because of incorrect hydrogen bonding between amino acids. 


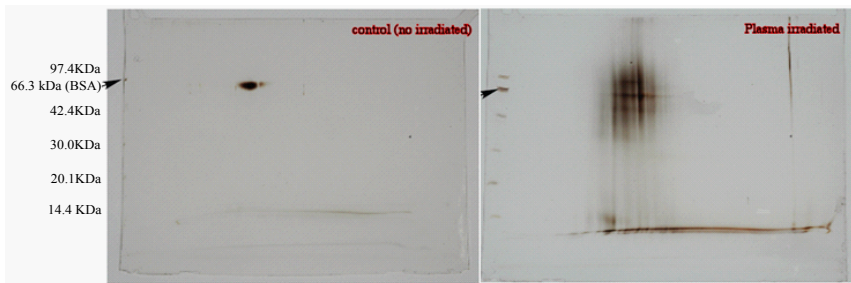

Figure 4: Deformation of bovine serum albumin (BSA) by nitrogen gas plasma which was confirmed by secondary electrophoresis.

Each arrow shows the original size of BSA. Compared with the one band of control BSA (left), exposed BSA by nitrogen gas plasma (right) is degraded and fragmentary. Some fragments may occur polymerization. Anyhow, deformation of BSA is occurred by nitrogen gas plasma.

Material: Bovine serum albumin (BSA) $500 \mathrm{ng}$

Method: Secondary SDS Poly-Acrylamide Gel Electrophoresis

Low pressure nitrogen gas plasma reactor: Anode-Cathode Gap: 40mm, Vac: $43000 \mathrm{~Pa}$, nitrogen gas flow speed: $6 \mathrm{~L} / \mathrm{min}$, Time: $30 \mathrm{~min}$, Temperature: $60^{\circ} \mathrm{C}$ (Temperature was measured inside of plasma) (Electric Source) Input Power: 84W, Rep. Freq. 2.5kHz, Peak Voltage:19.0 kV.

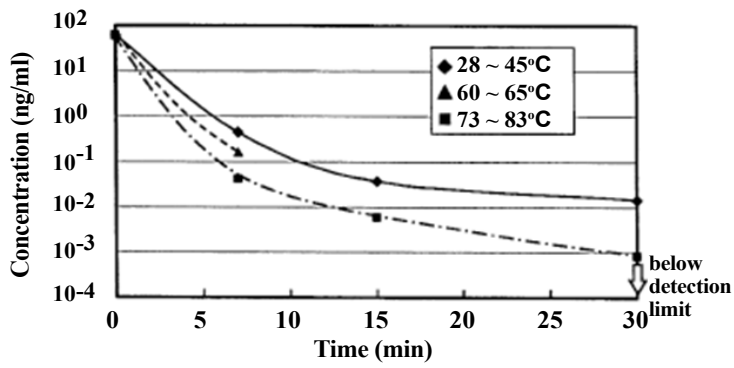

Figure 5: Temperature dependence of endotoxin inactivation by low pressure nitrogen gas plasma exposure.

Conditions of low pressure nitrogen gas plasma exposure: Input power, $84 \mathrm{~W}$ Frequency, 2.5 kHz; Temperature, 28-73 oC; Vaccum, 45 kPa; Gap, 40 mm; Flow rate, $15 \mathrm{~L} / \mathrm{min}$; Voltage, $19 \mathrm{kV}$.

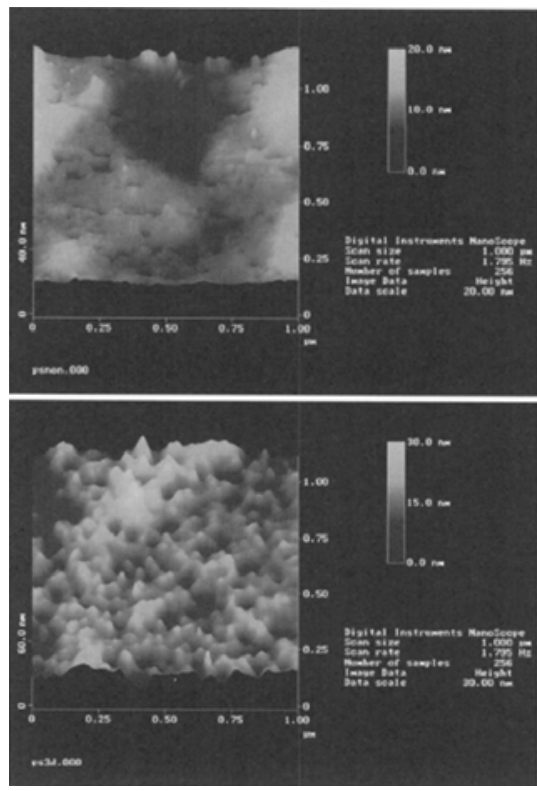

Figure 6: Surface analysis of Atomic Force Microscopy before and after nitrogen gas plasma exposure to polystylene.

Lower Figure: Before gas exposure (control); Upper Figure: After Gas Plasma treatment for 7 min by Low Pressure

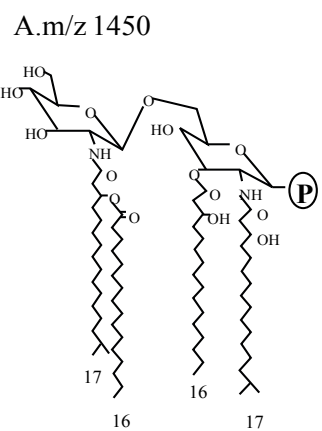

B.m/z 1435

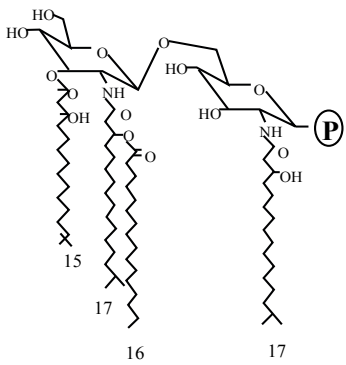

Figure 7: Chemical structure of $\mathrm{m} / \mathrm{z} 1450$ and 1435, respectively. They are produced by cleavage of esterbonding.

USFDA requires at least three log reductions of endotoxin (FDA Guidance for Industry, 2004) and dry heating at $250^{\circ} \mathrm{C}$ for $30 \mathrm{~min}$ is recommended, but almost all polymer materials cannot withstand by this procedure. Three logarithm reductions can be achieved by using nitrogen gas plasma exposure for $10 \mathrm{~min}$ at $28-45^{\circ} \mathrm{C}$ without any deterioration of materials (Figure 5) [6]. More than $5 \log$ reduction of endotoxins was completed by nitrogen gas plasma exposure at $73-83^{\circ} \mathrm{C}$ for $30 \mathrm{~min}$ together with material and functional compatibility and $10^{-6} \mathrm{SAL}$. Inactivation of endotoxin can be conducted by the ester bond cleavage of fatty acid of Lipid A [9], active site of endotoxin, by $\mathrm{OH}$ and/or $\mathrm{NO}$ radicals produced from nitrogen gas plasma exposure. As a result, m/z of 1450 and/or 1435, which cleaved one ester bonding of Lipid A of $E$. coli respectively, was identified. The pyrogenic activity of $\mathrm{m} / \mathrm{z}$ of 1450 and 1435 was confirmed to be lost (Figure 7) [10].

From these results, we can confirm nitrogen gas plasma exposure is effective to attain prion and endotixin inactivation. In addition both SAL of $10^{-6}$ and material/functional compatibility can be successfully achieved.

\section{References}

1. Fichet G, Comoy E, Duval C, Antloga K, Dehen C, et al. (2004) Novel methods for disinfection of prion-contaminated medical devices. Lancet 364: 521-526.

2. McDonnell GE (2007) Mechanisms of Prion Resistance in Antisepsis, Disinfection, and Sterilization. ASM Press Washington DC: 318-320.

3. Taylor DM (2004) Transmissible degenerative encephalopathies: inactivation of the unconventional causal agents, in Principles and Practice of Disinfection Preservation and Sterilization, Fraise AP, Lambert PA, Maillard JY (eds) Blackwell publishing, Oxford, UK: 324-344.

4. Fairand BP (2002) Radiation Sterilization for Healthcare Products. New York CRC Press: 39-41.

5. Halliwell G, Gutteridge J (2007) Free Radicals in Biology and Medicine. Oxford University Press, New York: 222-229.

6. Shintani H, Shimizu N, Imanishi Y, Sekiya T, Tamazawa K, et al. (2007) Inactivation of microorganisms and endotoxins by low temperature nitrogen gas plasma exposure. Biocontrol Sci 12: 131-143. 
Citation: Shintani H (2012) Inactivation of Prion and Entotoxins by Nitrogen Gas Plasma Exposure. Pharm Anal Acta 3:177. doi:10.4172/21532435.1000177

Page 4 of 4

7. Shintani $H$, McDonnell $G$ (2011) Inactivation of microorganisms (spore types and vegetative cells) and the mechanism by gas plasma in Sterilization and Disinfection by Plasma. Sakudo A, Shintani H (eds) Nova Science Publishers, New York: 33-48.

8. McDonnell GE (2007) Plasma in Antisepsis, Disinfection, and Sterilization. ASM Press, Washington DC: $184-186$
9. Williams LK (2007) Microbial Biodiversity and Lipopolusaccharide Heterogenicity: from Static to Dynamic Models in Endotoxins, Pyrogens, LAL Testing and Depyrogenetion. Informa Healthcare, New York: 111-131.

10. Darveau RP, Pham TT, Lemley K, Reife RA, Bainbridge BW, et al. (2004) Porphyromonas gingivalis lipopolysaccharide contains multiple lipid A species that functionally interact with both toll-like receptors 2 and 4 . Infect Immun 72 5041-5051. 